\title{
JÓVENES EN LA ENCRUCIJADA
}

JUAN CORNEJO E.

\begin{abstract}
RESUMEN
El acompañamiento de construcción de identidades de jóvenes homosexuales implica particular complejidad en consideración al carácter obligatorio con que se reviste la heterosexualidad y al hecho de que la escuela se ha constituido en guardiana y reproductora del orden heterosexista. El problema, sin embargo, se presenta cuando algunos(as) jóvenes se resisten a ese orden. En esencia, una comunidad LGBT es una comunidad de diferencias, unificadas por el hostigamiento, intolerancia y discriminación de que son objeto sus miembros. El círculo de violencia se debilita en la medida en que los propios sujetos, víctimas de esa violencia, deciden «salir del clóset»; de lo contrario, corren el riesgo de convertirse en rehenes del mismo. En el contexto escolar, los elementos que pueden ayudar en ese proceso son: la ruptura de la cultura del silencio, evitar el bullying antihomosexual y la promoción de redes de apoyo y de soporte de pares, escuela y familia.
\end{abstract}

PALABRAS CLAVE: ESCUELA, IDENTIDAD SEXUAL, «SALIR DEL CLÓSET»

* Doctor en Ciencia Política e Historia; Postdoctorando de la Facultad de Humanidades de la Universidad de Santiago de Chile (USACH).

Correo electrónico: jcornejoespejo@yahoo.es. 


\title{
JOVENS NA ENCRUZILHADA
}

\begin{abstract}
RESUMO
O acompanhamento da construção de identidades de jovens homossexuais apresenta complexidade específica devido à heteronormatividade e ao fato de que a escola se constitui guardiã e reprodutora da ordem heterossexista. O problema, todavia, ocorre quando alguns/mas jovens resistem a esta ordem. Essencialmente, uma comunidade LGBT é uma comunidade de diferenças, unida pela hostilidade, intolerância e discriminação sofridas por seus membros. O círculo de violência se enfraquece à medida que os próprios sujeitos, vítimas desta violência, decidem «sair do armário»; do contrário, correm o risco de tornarem-se reféns da situação. No contexto escolar, os elementos que podem colaborar nesse processo são a ruptura da cultura do silêncio, prevenção do bullying anti-homossexual e a promoção de redes de apoio e suporte de pares, escola e família.
\end{abstract}

PALAVRAS-CHAVE: ESCOLA, IDENTIDADE SEXUAL, «SAIR DO ARMÁRIO»

\section{YOUTH AT THE CROSSROADS}

\begin{abstract}
The accompanying construction of gay youth identity is particularly complex, given that compulsory heterosexuality is coated and the fact that the school has become the guardian and reproductive heterosexist order. The problem, however, occurs when some (as) young people are resisting that order. In essence LGBT community is a community of differences, unified the harassment, intolerance and discrimination that they experience its members. The circle of violence is weakened to the extent that the subjects themselves, victims of violence, decided to "come out», otherwise they risk becoming hostage to it. In the school context elements that can help in this process are: the breakdown of the culture of silence, preventing bullying and promoting antinohosexual support networks and support: peers, school and family.
\end{abstract}

KEY WORDS: SCHOOL, SEXUAL IDENTITY, «COMING OUT OF THE CLOSET» 


\section{INTRODUCCIÓN}

HISTÓRICAMENTE, LA ESCUELA HA sido productora de diferencias, distinciones y desigualdades. A través de múltiples mecanismos, ha clasificado y ordenado. Concebida inicialmente para acoger a algunos, lentamente fue procurada por aquellos(as) a los(as) cuales había sido negada. Los nuevos grupos, ineludiblemente, fueron introduciendo transformaciones en la institución. Para mantener las distinciones, ella hubo de promover otros elementos que garantizasen, explícita o implícitamente, las diferencias entre los sujetos. Sirviéndose de símbolos y códigos, ella afirma lo que cada uno puede o no puede hacer, señalando a aquellos(as) que deberán ser modelos y en los(as) cuales los sujetos pueden proyectar sus ideales de vida.

A este respecto, Foucault (1997), en su célebre texto: Vigilar y castigar, sostiene que el poder toma a los individuos, al mismo tiempo, como objetos y como instrumentos. Así, el proceso de «fabricación» de los sujetos es continuado y generalmente muy sutil, casi imperceptible. De allí que en las prácticas cotidianas, en los gestos, en las palabras banales, etcétera, se ha de poner especial cuidado; es decir, desconfiar de lo considerado «natural». Debemos atender no sólo a lo que enseñamos, sino al modo como lo hacemos y a los sentidos que nuestros alumnos(as) le dan a lo que aprenden. Tenemos que estar especialmente alertas a nuestro lenguaje, procurando percibir la homofobia, el sexismo, el racismo, el etnocentrismo, etcétera, que frecuentemente conlleva y funde.

Pero el lenguaje instituye y demarca los lugares de los géneros no sólo ocultando lo femenino, sino también con las adjetivaciones atribuidas a los sujetos, por el uso (o no) del diminutivo, por la elección de los verbos, por las asociaciones y por las analogías hechas entre determinadas cualidades, atributos, comportamientos y géneros. Además, tan o más importante que escuchar lo dicho sobre los sujetos es captar lo no dicho, lo silenciado, los sujetos que no son, ya sea porque no pueden asociarse a los atributos deseados, ya sea porque no pueden existir por no poder ser nominados. Probablemente, nada más ilustrativo del ocultamiento y negación de los(as) homosexuales/lesbianas, y de la homosexualidad en general, que el sistema escolar. No sin razón, antes en los ambientes religiosos se hablaba del «pecado innominable».

Siendo ese el ambiente que rodea a la homosexualidad en el contexto escolar, nos proponemos en las siguientes páginas reflexionar acerca de la necesidad de romper con la "cultura del silencio» — generadora de violencia y exclusión - imperante en la escuela en relación al tema, y 
llamar la atención acerca de la necesidad de respetar, acompañar y contener a aquellos(as) jóvenes que tempranamente deciden «salir del clóset» (coming out), de modo de aminorar los costos psíquicos y emocionales que demanda ese proceso. Asimismo, favorecer y promover escuelas «inclusivas» que, más que un problema, ven en las diferencias, cualesquiera que sean, una oportunidad de crecimiento y humanización.

\section{LA HETEROSEXUALIDAD OBLIGATORIA}

Uno de los tópicos más complejos de tratar en el ámbito educacional es el de la homosexualidad, no sólo por las restricciones, mitos y fantasías sociales que existen sobre el asunto, sino principalmente por los desafíos que supone el acompañamiento del proceso de construcción de la identidad sexual de un(a) joven que durante la adolescencia, o aun en la más temprana infancia, comienza a descubrirse diferente en relación con los(as) demás. Este proceso se torna particularmente complejo tanto para la persona implicada en el asunto, cuanto para quienes le rodean, en consideración a la obligatoriedad con que se reviste la heterosexualidad. Es lo que Friend (1993) apunta como: «la creencia de que todos son o deberían ser heterosexuales». Esta misma idea ya había sido manifestada varios años antes por Rich (1980) en su artículo: «La heterosexualidad obligatoria y la existencia lesbiana».

Basados en la presunción universal de la heterosexualidad y el consecuente régimen de privilegios, se establecen puniciones sociales para quien se aparta de esta norma. Así, el heterosexismo se constituye en un prejuicio institucionalizado en contra de la homosexualidad, mantenido por el consenso social que sanciona y promueve esta ideología (Friend, 1993:211). Idénticamente, Lorde (1985) describe el heterosexismo como: «la creencia en la inherente superioridad de un patrón amoroso sobre todos los otros, y el consecuente derecho de dominación» (Lorde, 1985:3). Es decir, la hegemonía de la ideología heterosexista, ${ }^{1}$ necesariamente legitima y promueve la homofobia, la cual es descrita como el terror que circunda a los sentimientos de amor hacia personas del mismo sexo (Lorde, 1985:4).

1 Esta hegemonía en el contexto universitario ha sido definida como «heterosexualidad compulsoria» por Eyre (1997) en el libro: Radical In(Ter)Ventions. Identity, Politics, and Difference/s in Education Praxis. Y como «heterosexualidad compulsiva» en el ámbito de la educación secundaria por Pascoe (2007) en el libro: Dude you're a fag. Masculinity and Sexuality in High School. 
Friend describe la homofobia como el temor y odio hacia la homosexualidad propia o de los otros (1993:211). Fone (2000), por su parte, señala que el término homofobia ha sido construido para significar el temor y aversión que provoca la homosexualidad y aquéllos que la practican. ${ }^{2}$

De esta forma, el «salir del clóset», y el subsecuente proceso de visibilización pública, resulta crucial para la lucha política y cultural de eliminación del heterosexismo y la homofobia. Tal proceso necesariamente debería tener como consecuencia no sólo la resistencia al orden heteronormativo sino su deconstrucción, como sostienen DePalma y Jennett (2007) en el libro: Challenging Homophobia: teaching about Sexual Diversity.

\section{EL LENGUAJE, CREADOR DE IDENTIDADES INDIVIDUALES Y COLECTIVAS}

El lenguaje cambia y su significado está estrechamente vinculado a los contextos sociales y culturales. Lo mismo se puede decir del término «identidad», el cual describe lo que se es en un momento dado, lo que se creía o se aspira a ser (Hall, 1990). En esta línea se puede analizar, en el contexto anglosajón, el término «queer», que puede ser usado para especificar a las personas que se identifican a sí mismas como lesbianas, gays o bisexuales; sin embargo, no todos concuerdan con esta palabra en su definición personal. Lo mismo se puede decir en relación con el vocablo «gay», el cual caracteriza a aquella persona identificada (o preferentemente identificada) con la atracción erótico-afectiva que despiertan individuos del mismo sexo. No obstante, muchos bisexuales, hombres y mujeres, no se identifican a sí mismos como «gays». Es decir, lesbiana, gay, bisexual, queer u otra palabra son designaciones que intentan dar cuenta de realidades y percepciones diferentes. En décadas pasadas, y aún hoy en día, en algunos países del primer mundo se ha privilegiado el término «queer», dado el potencial político y cultural de cambio que él representa, sin tomar en cuenta el carácter trasgresor de la heteronormatividad de que se le ha dotado.

En consideración a los antecedentes anteriormente expuestos, hemos de subrayar el carácter creador de identidades individuales y colectivas de que goza el lenguaje, lo cual no desconoce la movilidad

2 Para mayor información acerca de la homofobia consultar: Blumenfeld (1992); Caro; Guajardo (1997); Borrillo (2001) y Tin (2008). 
y resignificación de los términos. En este sentido, la postmodernidad no sólo ha reconocido el rol del lenguaje en el modelamiento individual y colectivo de la identidad, sino que también ha destacado su carácter performativo. En definitiva, los códigos culturales, los esquemas de percepción, los valores, las jerarquías de las prácticas establecidas, etcétera, reproducen lo que la sociedad establece con carácter de deseable y bueno. De este modo, la norma heterosexual aparece como un código que ha sido pasado de generación en generación a través de las instituciones sociales; entre otros, la escuela.

La teoría de la reproducción social evidencia el modo en que los patrones de dominación cultural han sido reconstruidos en generaciones subsecuentes. La escuela representa uno de los focos principales, al punto de constituirse en guardiana y reproductora, en este caso, del orden heterosexista. Con todo, el problema se presenta cuando los(as) jóvenes se resisten consciente o inconscientemente a ese orden, ya sea a través de sus comportamientos y actitudes, ya sea por sus deseos o sentimientos velados o manifiestos. La resistencia, en este sentido, aparece como la respuesta a las fuerzas culturales impositivas que pretenden instaurar un modelo, normas, valores o aun identidades únicas, excluyentes de cualquier otra.

\section{COMUNIDAD LGBT: COMUNIDAD DE DIFERENCIAS}

En esencia, se puede afirmar que una comunidad LGBT es una comunidad de diferencias; no sólo porque se halla traspasada por otras variables: edad, origen étnico, extracción socioeconómica, nivel educacional, etcétera, sino porque entre los sujetos implicados se pueden evidenciar distintas aspiraciones o intereses. Sin embargo, lo que aparentemente une a este grupo disímil y diverso es la pertenencia (o sensación de pertenencia) a un grupo marginalizado y reprobado socialmente. Esta situación hace que surja una suerte de solidaridad entre todos(as) ellos(as) dada su condición de marginalidad, pasando a un segundo plano los otros contrastes, o tornándolas irrelevantes.

En este contexto, la identidad resulta ser el sentido de sí mismo que emerge de la interacción entre lo individual y la experiencia social (Mead, 1934), en el cual la cultura actúa como marco referencial de la socialización. Tal noción clásica de cultura se desprende de las afirmaciones de quien sostuvo que el ser humano es un animal que está obligado a proveer significados de sí mismo. Todavía más, la cultura, según McLaren (1991), en ambos casos constituye el medio y el resul- 
tado de los discursos. Por otra parte, a la cultura no sólo la podemos asociar a un metafenómeno que existe a un nivel societal o en ninguna parte, porque necesariamente está presente en las instituciones u organizaciones sociales exhibidas como únicas.

Numerosos estudios evidencian que en todo el mundo, incluida Latinoamérica, los homosexuales aparecen como el primer grupo objeto de un odio social que muchas veces deviene en asesinatos no esclarecidos, mayoritariamente por falta de diligencia de las policías u órganos judiciales pertinentes y también por la omisión o vergüenza de los propios familiares de las víctimas, que optan por un cierre apresurado de los casos.

Sería esperable que ese clima de intolerancia no existiese o fuese menos agresivo en ambientes educativos, pero no parece así. Al igual que en otros ambientes sociales, la homofobia y el heterosexismo dejan sus marcas, al punto que no pocos estudiantes se han sentido violentados y vulnerados en sus derechos por el trato que reciben de parte de los(as) profesores(as) y del resto de sus compañeros(as).

El hostigamiento hacia los(as) jóvenes que desde temprano evidencian una orientación sexual distinta a la heterosexual se torna más visible en aquellos casos en que el disimulo, el ocultamiento, la invisibilización («no lo diga», «no lo externalice») se hace más complejo o aun imposible. Por ejemplo, los(as) estudiantes que desde sus primeros años de vida se identifican con el género opuesto al que le corresponde a su sexo biológico. No resulta extraño, entonces, que estos(as) estudiantes, salvo rarísimas excepciones, se vean obligados(as) a abandonar prematuramente el sistema escolar formal, y que por eso evidencien baja escolaridad y tasas que doblan o incluso triplican el analfabetismo del resto de la población.

No menos problemático resulta para profesores, directivos y encargados de reparticiones públicas de educación lidiar con este tipo de jóvenes, no sólo por la complejidad de las situaciones a las que deben dar respuesta $-\mathrm{y}$ para las cuales no se sienten preparados-, sino también porque son parte, a través de un universo valórico, de creencias, convicciones, etcétera, de cierto orden heterosexista, excluyente, por definición, de cualquier conducta, actitud o comportamiento que aparezca como trasgresor del mismo. Es más, a los educadores, quienes por su función de transmisores de conocimientos, valores, creencias, cultura... además aparecen como garantes, se les responsabiliza, no pocas veces, de la perpetuación de ese orden. 
Particularmente incomprensible resultan las prácticas discriminatorias e intolerantes en los ambientes universitarios, donde se esperaría que no existiesen, en consideración a la necesaria apertura, crítica y antidogmatismo que deberían primar en la investigación, docencia y prácticas pedagógicas. Sin embargo, ello no siempre es así, como acusan numerosos autores.

\section{EL SIGNIFICADO DEL CRUCE DE FRONTERAS}

La oposición de paradigmas imperantes en la postmodernidad proporciona nuevos lenguajes a través de los cuales se hace posible la deconstrucción y cambio de las relaciones de dominación, poder y conocimiento legitimadas en las formas tradicionales del discurso.

Uno de los aspectos fundamentales, según sostienen numerosos autores, en el cambio de las relaciones de dominación estriba en que el sujeto con identidad no heterosexual asuma dicha realidad (el coming out); eso se torna más fácil cuando existe un grupo de referencia en el cual pueda elaborar dicha identidad. ${ }^{3}$ Grupo particularmente importante si se tiene en cuenta que la cultura heterosexista imperante tiende a invisibilizar o desacreditar tal posibilidad, al punto incluso de recurrir a la violencia como forma de desincentivar la construcción de una identidad que aparece discordante con el modelo hegemónico.

La relevancia de la visibilidad ha sido subrayada por Rhoads (1994) en sus análisis de lo que denomina «nacionalidad queer». Para este autor, el concepto queer rechaza la noción de que todos son iguales; vale decir, la idea central de lo queer es confrontacional y acentúa las diferencias como una manera de promover la propia identidad.

A este respecto, cabe recordar, como bien apuntó en su momento Foucault (2002), que la identidad homosexual no existía antes del siglo XIX. Pese a las discrepancias acerca del momento en que ella habría irrumpido en la historia, una cosa sí parece cierta: aunque la homosexualidad como identidad no habría existido antes de la medicalización de las prácticas homoeróticas (siglo XIX), los actos homogenitales, bajo diferentes denominaciones, tendrían presencia desde siempre en la historia de la humanidad.

3 La salida del clóset (el coming out) en el contexto escolar ha sido abordado por Sanlo (1998); Owen (1998); Baker (2002); Báez, Howd \& Pepper (2007); entre otros. 
No obstante, con el advenimiento del positivismo la homosexualidad adquirió un estatuto médico-psiquiátrico inédito. Catalogada desde ese momento una desviación, fue reducida a sus expresiones observables y medibles.

Detrás de estas consideraciones subyace el debate producido entre esencialistas y constructivistas. Para los primeros, entre los que se cuenta a los positivistas, es posible encontrar en la historia algunas constantes que permiten reconocer ciertas prácticas sexuales definibles en términos de identidad; para los segundos, en cambio, si bien se pueden reconocer algunas prácticas análogas en la historia, la identidad homosexual (o más recientemente gay) sólo es viable en el contexto de la cultura contemporánea. Un intento por superar aquello lo constituye la noción propuesta por Rhoads (1994): «gay ethnicity».

En este sentido, Foucault (1980) argumenta que la cultura es más que un mero subproducto de la vida social. La cultura divide la vida social, al punto de que los discursos juegan un rol fundamental en el entendimiento y modelamiento de la misma. Él analiza conocimiento y verdad en términos de bases para la institucionalización de los mecanismos de control, y como recursos para la exclusión de las conductas desviantes de la vida social.

La medicalización de la homosexualidad no persiguió otro objetivo que el de reemplazar el castigo físico -imperante hasta el siglo XVIII- aplicado bajo el paradigma criminalizador que diseñó y promovió la sociedad capitalista-burguesa, por la responsabilidad del ciudadano. En este contexto, la heterosexualidad irrumpió como elemento «normalizador» y de vigilancia de la vida social. Todo aquel que se alejaba de sus dictados fue considerado un enfermo o, en el mejor de los casos, un subversivo del orden heterosexista.

Todo este discurso y consecuente representación de lo considerado «normal», «sano» o «deseable» se revistió de una cientificidad transformada en régimen de verdad. Y precisamente esa pretensión ha sido uno de los ejes focales de la crítica postmoderna, pues no sólo se reclama un conocimiento basado en el conocimiento científico empirista, sino también uno sustentado en la subjetividad y la experiencia personal; es decir, el conocimiento científico no representa la totalidad del conocimiento (Lyotard, 1984). Dicho en otros términos, la crítica postmoderna denuncia precisamente aquella pretensión «normalizadora» de un cierto tipo de ciencia que medicalizó la homosexualidad. En el fondo, se trata de un poder que ha definido la heterosexualidad como la norma y la homosexualidad como la desviación. 
El poder se revela a través del predominio de ciertas ideologías sobre otras, con las consecuentes ventajas para aquel grupo que adhiere a la misma, en contraposición a aquellos que están en una posición desmejorada. A menudo, los grupos de poder ejercen su primacía por medio de estrategias sutiles, entre las que se cuentan sus narrativas de conocimiento, sus discursos y su producción cultural legitimada e institucionalizada a través de una multiplicidad de mecanismos.

Siendo esto así, no nos debe extrañar, entonces, que en las escuelas la heterosexualidad aparezca con la categoría de norma inobjetable, mientras la experiencia homosexual se halle necesariamente desacreditada y excluida de la vida escolar. En contraposición a esta realidad, Gergen (1991) describe la postmodernidad a la manera de un mercado donde se proyecta una multiplicidad de voces que aspiran a ser aceptadas y legitimadas como expresiones de verdad. La pregunta que surge de inmediato: ¿estarán las escuelas preparadas para aceptar y promover sus propias diferencias internas o, por el contrario, la homosexualidad no es más que una de las tantas expresiones de anquilosamiento que asfixia la promoción de la diversidad en todas sus formas y manifestaciones?

Lo anterior resulta sumamente ilustrativo, en especial si se tiene en cuenta que la crítica postmodernista busca tender un puente entre la investigación y la acción impuesta por el positivismo; o sea, su objetivo no es otro que el de restablecer el relacionamiento entre investigación y praxis. En lo que dice relación con el proceso de autodefinición de un sujeto no heterosexual, aquello resulta del todo crucial por cuanto el propio individuo debe definir lo que implica «salir del clóset» (el coming out). Él define los medios, los tiempos y los recursos que supone ese proceso de liberación personal. Él también ha de evaluar los obstáculos que le impone el sistema. No se debe olvidar, asimismo, que es la sociedad dominante, por medio de la escuela, la que se sirve de ella para imponer sus restricciones.

\section{MARGINACIÓN Y EMPODERAMIENTO}

La resistencia a ese intento de subyugamiento proviene, precisamente, de la propia subcultura gay juvenil, que en este caso aparece como la resistencia a la dominación de la organización social. Otros autores prefieren el término contracultura, en el entendido de la interacción que define la colectividad en oposición a la cultura dominante, que comprende, entre otros aspectos, valores, creencias y actitudes. 
Nuehring, Beck y Tyler (1989) examinaron la estructura informal de la comunidad gay y lesbiana y concluyeron que el concepto contracultura comprende, básicamente, los elementos que evidencian el sistema de preferencias sexuales de dicha comunidad, basado en normas que se hallan en conflicto con los valores de la mayor parte de la sociedad. Esta existencia sólo puede ser entendida de acuerdo a la colisión con la cultura dominante.

El conflicto que se suscita para aquellos jóvenes que buscan el reconocimiento de su identidad y, en consecuencia, de sus derechos al interior del sistema educacional es, precisamente, que en ese afán pierdan el sentido de la diferencia («queerness») con la que ellos se definen a sí mismos. En otras palabras, y como ha ocurrido con otras comunidades en similar situación, siempre está presente el riesgo de ser cooptados por el sistema que combatían. Este es, probablemente, el mayor desafío que enfrentan estos jóvenes: evitar su absorción por el sistema, pero sin renunciar a la reivindicación de sus derechos.

Los elementos protectores de este eventual riesgo serían, por ejemplo, el mantenimiento de su condición de «comunidades de diferencia» y los lazos de solidaridad y reciprocidad que se puedan establecer entre los propios gays y lesbianas; sin embargo, en este último caso se presenta el problema de que no todos los estudiantes evidencian el mismo grado de envolvimiento, compromiso e identificación con el proceso liberador («Outness»). De allí la necesidad de integrar a un número creciente de individuos en un proceso que persigue la propia realización personal. En el ámbito del sistema educacional, en vista de la diversificación y ampliación de conocimientos, una alternativa viable y necesaria consiste en la creación de espacios en la academia para los estudios gay-lésbicos.

\section{REHENES DEL CLÓSET}

El clóset simboliza la opresión de que han sido objeto lesbianas, gays y bisexuales, quienes se han visto forzados a silenciar su propia identidad sexual. «El clóset es la estructura que representa la opresión gay en este siglo» (Sedgwick, 1990: 68). La normalización de la sexualidad ha creado y continúa reforzando el clóset, dado que la heterosexualidad ha sido, en parte, ordenada a través del discurso. Contrariamente, la homosexualidad ha sido presentada como un acto aberrante o, en el mejor de los casos, algo invisible e inmencionable. 
Estas apreciaciones impuestas al sentido común de nuestra cultura han creado un clima de temor y vergüenza para aquel que osa traspasar los estrechos márgenes del gueto autoimpuesto, al punto que los propios sujetos homosexuales se han convertido en guardianes de ese orden de exclusión. El sentimiento de rechazo y temor a los rumores con los que puedan verse expuestos públicamente operan cual mejor dispositivo de control y autocensura. Ahora bien, cuando estos recursos resultan insuficientes, siempre queda la posibilidad de recurrir a la violencia en sus más variadas formas, donde la verbal ocupa en este arsenal disuasivo un lugar de privilegio.

Muchos padres de jóvenes homosexuales tampoco escapan a este ordenamiento disciplinador. Con sus chantajes emocionales, no pocas veces los obligan a cercenar sus expresiones erótico-afectivas, con el consecuente daño en su autoestima. Otro de los efectos de este enclaustramiento identitario es la alta tasa de suicidios. El suicidio ronda como una posibilidad cuando comienzan a descubrirse gays o lesbianas. Muchos de ellos ven en esta drástica medida la única salida para acabar con su sufrimiento y para terminar definitivamente con una atracción que les parece prohibida. Por su parte, los que sobreviven deben lidiar permanentemente con el temor y resentimiento asociados a su orientación sexual, en especial en países donde la homofobia cultural se halla institucionalizada.

En este universo de creencias, las ideas de desvío, anormalidad y patología promovidas por una sexualidad medicalizada no hacen sino reforzar la fuerza del clóset. De manera permanente se buscan las causas remotas o la etiología de la homosexualidad para la exculpación. A esta búsqueda obsesiva, sin duda, contribuyen muchos investigadores que ingenuamente insisten en hallar la génesis de la homosexualidad usando como modelo de referencia incuestionado y jamás sometido a análisis la heterosexualidad. No perciben que tras esta búsqueda hay un referente ideológico no explicitado, pues más que preguntarse por su origen sería más prudente ahondar en la multiplicidad de expresiones que asume la sexualidad humana.

Lo sorprendente de todo es que el homosexual permanentemente se siente impelido por diferentes instancias sociales (familia, escuela, etcétera) a preguntarse por el origen de su orientación, mientras el heterosexual jamás se ha cuestionado las motivaciones de su inclinación. La heterosexualidad, en este contexto, se sitúa como una premisa a priori de normalidad. De allí que no sea extraño, entonces, que muchos homosexuales ocupen bastante espacio en sus vidas intentando 
entenderse a sí mismos. Pero una cosa que parece indesmentible, tanto en países más abiertos y tolerantes, cuanto en aquellos donde la cultura del silencio impone sus reglas, es que, respecto de décadas pasadas, a las generaciones más jóvenes les toma menos tiempo y con menos traumas y secuelas psicológicas salir del clóset.

Un factor decisivo en la facilitación de este proceso ha sido el hecho de que la homosexualidad, pese a las restricciones sociales y censuras provenientes de los grupos conservadores, se ha convertido en un tema ineludible en razón del VIH/SIDA. Para estos jóvenes, la autorrevelación puede ocurrir ya sea por un malestar interno que les demanda mayores dosis de honestidad consigo mismos, ya sea porque las personas que los rodean los impulsan a aquello; o ambos estímulos simultáneamente.

Resulta fundamental el apoyo de la familia en términos de sanidad mental y soporte emocional. Sin lugar a dudas, aquellos jóvenes que cuentan con una familia que los acoge y comprende en el proceso de asumirse, además de encontrarse más preparados para resistir las presiones sociales y la homofobia cultural, no ven lesionada su autoestima; sin contar que están en mejores condiciones para prevenir el $\mathrm{VIH} / \mathrm{SIDA}$ o cualquier otra ITS, dado que cuentan con mayores redes de apoyo y condiciones de autoprotección.

Una sociedad que relega a las personas LGBT al clóset, además de inhibir las expresiones erótico-afectivas decantadas en proyectos de pareja, fomenta el sexo descomprometido y casual. Ello trae como consecuencia el riesgo de contraer el VIH/SIDA, no sólo por las condiciones en que se producen los encuentros sexuales furtivos, sino porque debido a la baja autoestima muchas de esas personas recurren a estas prácticas sin los debidos resguardos, ante el autoconvencimiento, incentivado por la homofobia internalizada, de que son incapaces de establecer verdaderas relaciones de amor y compromiso.

Evitando caer en moralismos descarnados que se alojan en el propio lenguaje que califica las prácticas homoeróticas de promiscuas, resulta evidente que el sexo casual, la pornografía u otras prácticas, donde la autoprotección y el cuidado del otro son evadidos, en sociedades profundamente homofóbicas aparecen como las únicas salidas para personas con una orientación distinta a la heterosexual. Sin contar que el sexo anónimo, eventualmente, podría contribuir a reforzar los cerrojos del clóset, al dificultar los relacionamientos amorosos entre personas del mismo sexo (Rhoads, 1994). 
Una sociedad homofóbica, lejos de crear condiciones de protección, expone innecesariamente a las personas LGBT al VIH/SIDA, a través de la culpabilización, la humillación, la burla, el desprecio... Otro riesgo no menos impactante al que las expone al inhibirles su autorrealización y confinarlas al gueto es la exposición a situaciones de violencia física, abusos o extorsiones; inclusive, en muchos casos, de parte de los propios agentes de seguridad del Estado.

En el ámbito escolar, un papel clave en la construcción identitaria podría ser cumplido por los grupos de pares al interior de los propios establecimientos educacionales, institutos profesionales o universidades, puesto que, junto con cumplir funciones de consejería, se podrían constituir en redes de apoyo, soporte y promoción del autocuidado.

\section{DESAFÍOS QUE ENFRENTA EL SISTEMA ESCOLAR PARA ABORDAR EL TEMA DE LA HOMOSEXUALIDAD}

En esta última parte, simplemente queremos esbozar algunos de los desafíos que el sistema escolar debería enfrentar para abordar la homosexualidad o, más específicamente, el de la diversidad sexual en su sentido más amplio. No es nuestra intención hacer un análisis pormenorizado de las distintas situaciones, pues ese esfuerzo, además de exceder los marcos de este artículo, requeriría por sí solo una investigación especial.

A nuestro entender, el primer gran desafío que debe enfrentar el sistema escolar es el silencio de las instituciones educativas ante una realidad que existe, por más que se pretenda ocultar. Romper con la cultura del silencio, sin embargo, no constituye una tarea que sólo compete a los(as) profesores(as) o a los padres de los(as) jóvenes homosexuales. Se hace necesario un trabajo multidisciplinario para entender un problema que, además de complejo, está rodeado de creencias, mitos, temores y prejuicios, no siempre fáciles de erradicar, pues la mayor parte de las veces están instaladas en la conciencia colectiva y en sus lógicas subyacentes.

En segundo término, se hace necesario adentrarse en la homofobia. Saber lo que es, cómo funciona y cómo se manifiesta en el sistema escolar. Para eso, resulta imprescindible estudiar las diversas expresiones que asume la sexualidad humana; la naturalización de las diferencias de género y la orientación sexual; las confusiones de género; los roles de género y la normatividad heterosexual. 
En tercer lugar, analizar acuciosamente las actitudes y visiones de la homosexualidad en el contexto escolar: lenguaje, estereotipos, transmisión del estigma homosexual, posibilidades de visibilidad o de blindaje del clóset, formas que asume la exclusión, subcategorías de la homofobia y otras discriminaciones transversales; además de los discursos institucionales. ${ }^{4}$

Como cuarta estrategia, una descripción pormenorizada de las vivencias personales de adolescentes homosexuales/lesbianas, a fin de discutir las posibilidades de inclusión, y reforzar las estrategias y redes de apoyo.

Quinto, resolver un problema no menor: la falta de formación y escasez de materiales educativos relativos al tema, especialmente dirigido para padres y educadores(as).

En sexto lugar, discutir las implicancias del impacto psicológico y emocional que produce el bullying antihomosexual en los(as) jóvenes con una orientación no heterosexual. En este sentido, se hace necesario enfrentar el tema de la construcción de la identidad LGBT en los(as) jóvenes, las consecuencias psicoemocionales de la homofobia, la alerta permanente ante las conductas autodestructivas o que merman la autoestima de estos(as) jóvenes; además del diseño de estrategias que eviten el hostigamiento, el miedo, el acoso y el bullying antihomosexual. ${ }^{5}$

Finalmente, proyectar escuelas inclusivas, donde el reconocimiento y respeto de las diferencias, cualesquiera que sean, no signifique un problema sino una oportunidad de crecimiento y humanización (Comisión educativa de COGAM, 2005).

SANTIAGO (CHILE), ABRIL 2010

RECIBIDO: MAYO 2010

ACEPTADO: JUNIO 2010

4 Ver el artículo de Peinado (2007): «Educación para la ciudadanía: ¿pensar la homosexualidad en clave educativa?».

5 A propósito de este tema ver Sears (2005); y Platero y Gómez (2007). 


\section{REFERENCIAS BIBLIOGRÁFICAS}

BAEZ, John; JeNNIFER Howd y RACHel PePPeR (2007): The Gay and Lesbian Guide to College Life. New York: Random House.

BAKER, J. M. (2002): How Homophobia Hurts children Nurturing Diversity at Home, at School, and in the Community. New York: Harrington Park Press.

BLUMENFELD, WARREN (1992): Homophobia: how we all pay the price. Boston: Beacon Press.

BORRILlO, DANIEL (2001): Homofobia. Barcelona: Ballaterra.

CARO, ISAAC y GABRIEL GUAJARDO (1997): Homofobia cultural en Santiago de Chile. Un estudio cualitativo. Santiago: FLACSO.

COMISIÓN DE EDUCACIÓN DE COGAM (2005). Homofobia en el sistema educativo. Madrid: COGAM.

DE PALMA, RENÉE y MARK JENNETT (2007): «Deconstructing heteronormativity in primary schools in England: cultural approaches to a cultural phenomenon». En LUTZ VAN DIJK y BARRY VAN DRIEL (ed.): Challenging Homophobia: teaching about Sexual Diversity. London: Trentham Books.

EYRE, LYNDA (1997): «Any more colorful we'd have to censor it». En Suzanne De Castell y Mary Bryson (ed.): Radical In(Ter)Ventions. Identity, Politics, and Difference/s in Education Praxis. Albany, New York: State University of New York Press.

FONE, BYRNE (2000): Homophobia: a History. New York: Picador USA. FouCAult, MiCHEL (2002). Historia de la sexualidad. Vol. 1. La voluntad de saber. Buenos Aires: Siglo XXI.

—_ (1980): Power/Knowledge. New York: Pantheon Books. (1997): Vigilar y castigar. México: Siglo XXI.

FRIEND, RICHARD (1993): «Beyond silenced voices: class, race and gender in United States school». En ROBERT RHOADS (org.): Choices, not closets: heterosexism and homophobia in School. Albany: State University of New York.

GERGEN, KenNeth (1991): The saturated self: Dilemmas of identity in contemporary life. New York: Basic Books.

LORDE, AUDRE (1985). I am your sister: Black women organizing across sexualties. Latham, NY: Kitchen Table Press, Women of Color Press.

LYOTARD, JEAN-FRANCOIS (1984): The postmodern condition. Minneapolis: University of Minnesota. 
HALL, STUART (1990): «Cultural identity and diaspora». En J. RUTHERFORD (ed.): Identity: community, culture, difference. London: Lawrence \& Wishart.

Mead, George Herbert (1934): Mind, self \& society. Chicago: University of Chicago.

MClAREN, PeTER (1991): «Decentering culture: postmodernism, resistance and critical pedagogy». En NANCY B. WYNER (ed.): Current perspectives on the culture of schools. Boston: Brookline Books.

NuEHRING, ELANE; SARA FEIN y MARY TYLER (1989): «The gay college student: perspective for mental health professionals». Counseling Psychologist 4 (4).

OWENS, ROBERT (1998): Queer Kids. The Challenges and Promise for Lesbian, Gay, and Bisexual Youth. New York: The Haworth Press.

PASCOE, C. J. (2007): Dude you're a fag. Masculinity and Sexuality in High School. Berkely: University of California Press.

PEINADO, MATILDE (2007): «Educación para la ciudadanía: ¿pensar la homosexualidad en clave educativa?». Revista de Antropología Experimental $\mathrm{N}^{\circ} 7$, Texto 16.

PLATERO, RAQUEL y EMILIO GÓMEZ (2007): Herramientas para combatir el bullying homofóbico. Madrid: Talasa.

RICH, ADRIANE (1980): «La heterosexualidad obligatoria y la existencia lesbiana». Signs: Journal of Women in Culture and Society 5, $\mathrm{N}^{\circ} 4$.

RHOADS, ROBERT (1994): Coming Out in College. The Struggle for a Queer Identity. Westport, Connecticut: Bergin \& Garvey.

SANLO, RONNI (1998) (ed.): Working with Lesbian, Gay, Bisexual, and Transgender College Students. Westport, Connecticut: Greenwood Press.

SEARS, JAMES (2005) (ed.): Gay, Lesbian and Transgender Issues in Education. Programs, Policies and Practices. New York: Harrington Park Press.

SEDGWICK, EVE (1990): Epistemología del armario. Barcelona: Ediciones de la Tempestad.

TIN, LOUIS-GEORGES (2008) (ed.): The dictionary of homophobia: a global history of gay \& lesbian experience. Vancouver: Arsenal Pulp Press. 